OPEN ACCESS

Edited by:

Alfredo Conti,

University of Bologna, Italy

Reviewed by:

Enricomaria Mormina

Università degli Studi di Messina,

Thomas Pic

Italy

Charité University Medicine Berlin,

Germany

*Correspondence:

Andrey Zhylka

a.zhylka@tue.nl

Nico Sollmann

nico.sollmann@tum.de

${ }^{t}$ These authors have contributed equally to this work and share first authorship

Specialty section:

This article was submitted to Neuro-Oncology and

Neurosurgical Oncology,

a section of the journal

Frontiers in Oncology

Received: 19 August 2021 Accepted: 15 November 2021 Published: 14 December 2021

Citation: Zhylka A, Sollmann N, Kofler F, Radwan A, De Luca A, Gempt J, Wiestler B, Menze B, Krieg SM, Zimmer C, Kirschke JS, Sunaert S, Leemans A and Pluim JPW (2021)

Tracking the Corticospinal Tract in Patients With High-Grade Glioma: Clinical Evaluation of Multi-Level Fiber Tracking and Comparison to Conventional Deterministic Approaches.

Front. Oncol. 11:761169. doi: 10.3389/fonc.2021.761169

\section{Tracking the Corticospinal Tract in Patients With High-Grade Glioma: Clinical Evaluation of Multi-Level Fiber Tracking and Comparison to Conventional Deterministic Approaches}

\author{
Andrey Zhylka ${ }^{1 * t}$, Nico Sollmann ${ }^{2,3,4,5 * t}$, Florian Kofler ${ }^{3,6,7}$, Ahmed Radwan 8,9 , \\ Alberto De Luca ${ }^{10,11}$, Jens Gempt ${ }^{12}$, Benedikt Wiestler ${ }^{3,7}$, Bjoern Menze $^{6,13}$, \\ Sandro M. Krieg ${ }^{4,12}$, Claus Zimmer ${ }^{3,4}$, Jan S. Kirschke ${ }^{3,4}$, Stefan Sunaert ${ }^{8,9,14}$, \\ Alexander Leemans ${ }^{10}$ and Josien P. W. Pluim ${ }^{1}$
}

${ }^{1}$ Biomedical Engineering, Eindhoven University of Technology, Eindhoven, Netherlands, ${ }^{2}$ Department of Diagnostic and Interventional Radiology, University Hospital UIm, Ulm, Germany, ${ }^{3}$ Department of Diagnostic and Interventional Neuroradiology, School of Medicine, Klinikum rechts der Isar, Technical University of Munich, Munich, Germany, ${ }^{4}$ TUM-Neuroimaging Center, Klinikum rechts der Isar, Technical University of Munich, Munich, Germany, ${ }^{5}$ Department of Radiology and Biomedical Imaging, University of California, San Francisco, San Francisco, CA, United States, 6 Image-Based Biomedical Modeling, Department of Informatics, Technical University of Munich, Munich, Germany, ${ }^{7}$ TranslaTUM - Central Institute for Translational Cancer Research, Technical University of Munich, Munich, Germany, ${ }^{8}$ Department of Imaging and Pathology, Translational MRI, Katholieke Universiteit (KU) Leuven, Leuven, Belgium, ${ }^{9}$ Department of Neurosciences, Leuven Brain Institute (LBI), Katholieke Universiteit (KU) Leuven, Leuven, Belgium, ${ }^{10}$ Image Sciences Institute, University Medical Center Utrecht, Utrecht, Netherlands, ${ }^{11}$ Neurology Department, UMC Utrecht Brain Center, University Medical Center Utrecht, Utrecht, Netherlands, ${ }^{12}$ Department of Neurosurgery, School of Medicine, Klinikum rechts der Isar, Technical University of Munich, Munich, Germany, ${ }^{13}$ Department of Quantitative Biomedicine, University of Zurich (UZ), Zurich, Switzerland, ${ }^{14}$ Department of Radiology, Universitair Ziekenhuis (UZ) Leuven, Leuven, Belgium

While the diagnosis of high-grade glioma (HGG) is still associated with a considerably poor prognosis, neurosurgical tumor resection provides an opportunity for prolonged survival and improved quality of life for affected patients. However, successful tumor resection is dependent on a proper surgical planning to avoid surgery-induced functional deficits whilst achieving a maximum extent of resection (EOR). With diffusion magnetic resonance imaging (MRI) providing insight into individual white matter neuroanatomy, the challenge remains to disentangle that information as correctly and as completely as possible. In particular, due to the lack of sensitivity and accuracy, the clinical value of widely used diffusion tensor imaging (DTI)-based tractography is increasingly questioned. We evaluated whether the recently developed multi-level fiber tracking (MLFT) technique can improve tractography of the corticospinal tract (CST) in patients with motor-eloquent HGGs. Forty patients with therapy-naïve HGGs (mean age: $62.6 \pm 13.4$ years, 57.5\% males) and preoperative diffusion MRI [repetition time (TR)/echo time (TE): 5000/78 ms, voxel size: $2 \times 2 \times 2 \mathrm{~mm}^{3}$, one volume at $b=0 \mathrm{~s} / \mathrm{mm}^{2}, 32$ volumes at $b=1000 \mathrm{~s} / \mathrm{mm}^{2}$ ] underwent reconstruction of the CST of the tumor-affected and unaffected hemispheres 
using MLFT in addition to deterministic DTI-based and deterministic constrained spherical deconvolution (CSD)-based fiber tractography. The brain stem was used as a seeding region, with a motor cortex mask serving as a target region for MLFT and a region of interest $(\mathrm{ROI})$ for the other two algorithms. Application of the MLFT method substantially improved bundle reconstruction, leading to CST bundles with higher radial extent compared to the two other algorithms (delineation of CST fanning with a wider range; median radial extent for tumor-affected vs. unaffected hemisphere - DTI: $19.46^{\circ}$ vs. 18.99,$p=0.8931$; CSD: $30.54^{\circ}$ vs. $27.63^{\circ}, p=0.0546$; MLFT: $81.17^{\circ}$ vs. $74.59^{\circ}$, $\mathrm{p}=0.0134)$. In addition, reconstructions by MLFT and CSD-based tractography nearly completely included respective bundles derived from DTI-based tractography, which was however favorable for MLFT compared to CSD-based tractography (median coverage of the DTI-based CST for affected vs. unaffected hemispheres - CSD: $68.16 \%$ vs. $77.59 \%$, $p=0.0075$; MLFT: $93.09 \%$ vs. 95.49\%; $p=0.0046$ ). Thus, a more complete picture of the CST in patients with motor-eloquent HGGs might be achieved based on routinely acquired diffusion MRI data using MLFT.

Keywords: fiber tractography, diffusion MRI, brain tumor, corticospinal tract (CST), neurosurgery planning

\section{INTRODUCTION}

Gliomas represent the most common malignant brain tumors in adults, with an average annual age-adjusted incidence rate of $\sim 4.67$ to 5.73 per 100,000 population $(1,2)$. Anaplastic astrocytoma and glioblastoma are the major high-grade glioma (HGG) entities and peak in elderly subjects $(1-3)$. Overall prognosis is poor, with a median survival below 2 years $(1,2,4)$. HGGs can be regarded as chronic progressive diseases and typically show infiltrative growth behavior, which renders curative treatment almost impossible for the majority of affected patients $(3,5)$.

Nowadays, the standard treatment approach in patients harboring HGGs is a combination of neurosurgical resection, extended focal radiotherapy, and adjuvant chemotherapy (6-9). Nonetheless, multiple factors including histopathological characteristics, molecular tumor biology, as well as functional eloquence of the affected brain region contribute to individual therapy decision-making in clinical practice $(9,10)$. Regarding neurosurgical resection, a maximum extent of resection (EOR) has been associated with prolonged survival rates and better quality of life (11-17). However, mostly depending on individual tumor location, achieving a maximum EOR can be in conflict with preserving specific functions, such as the ability to move or speak without constraints. Thus, the principle of contemporary brain

Abbreviations: 3D, three-dimensional; BraTS, Brain Tumor Segmentation; CSD, constrained spherical deconvolution; CST, corticospinal tract; DES, direct electrical stimulation; DTI, diffusion tensor imaging; EOR, extent of resection; FA, fractional anisotropy; FOD, fiber orientation distribution; FLAIR, fluid attenuated inversion recovery; HGG, high-grade glioma; MLFT, multi-level fiber tracking; MNI, Montreal Neurological Institute; MRI, magnetic resonance imaging; PACS, Picture Archiving and Communication System; ROI, region of interest; nTMS, navigated transcranial magnetic stimulation; SD, standard deviation; TFE, turbo field echo; TE, echo time; TR, repetition time; VBG, Virtual Brain Grafting; WM, white matter. tumor surgery aims at an optimum EOR whilst avoiding surgeryrelated functional decline as far as possible $(18,19)$.

The gold-standard method for spatially resolved assessment of brain function is intraoperative direct electrical stimulation (DES), which can be applied as a strategy to guide neurosurgical resection and to avoid functional deficits in the course of tumor resection $(8,20-22)$. In addition to intraoperative DES, presurgical imaging is paramount to achieve an optimized onco-functional result. At the forefront of imaging techniques, multi-sequence magnetic resonance imaging (MRI) is applied to gather insights into spatial location, spread, and phenotyping of brain tumors (23-26). Lately, diffusion tensor imaging (DTI) in particular has seen increasing relevance as it allows identifying and delineating subcortical white matter (WM) structures non-invasively (27-31). In the neurosurgical context, DTI is frequently used for tracking of the corticospinal tract (CST), the main WM pathway subserving human motor function. The popularity of DTI can be explained by the low false-positive rate of tractography maps (32). Yet, the approach tends to produce underrepresented fiber bundles (33). Consequently, this causes an ongoing debate on whether conventional DTI methods are accurate and reliable enough to serve as a workable solution for delineating WM architecture in patients with glioma (34-37). Specifically, one main criticism is that the brain's WM architecture harbors numerous fiber crossings and further complex geometrical configurations, including fiber branching, which are hard to resolve $(38,39)$.

While deterministic tractography with DTI is the most common preoperative approach, a variety of models has been proposed to overcome the overall limitations of DTI-based tractography and improve the reconstruction of WM fiber organization as an attempt to further narrow the gap between imaging and reality, including diffusion kurtosis imaging and fiber orientation distribution (FOD)based approaches using constrained spherical deconvolution (CSD) as the most prominent representative (40-42). At the same time, although these models are more capable of disentangling fiber 
orientations, tractography algorithms commonly impose additional constraints that are set to achieve structurally plausible results, such as angular deviation and consideration of all the orientations as separate fibers. Consequently, either pathway propagation may be terminated, or a diverging branch may be pruned due to the angular limitations as well as related to spatial resolution constraints, which may artificially push the rate of false-negative findings (43). Thus, the possibility of incorporating fiber bifurcations with high angular deviations, such as those observed for the human CST, remains neglected. Probabilistic algorithms that are supposed to improve reconstructions by not just propagating into the peak FOD direction but sampling each step from the FOD could compensate for the angular resolution of the FOD model and capture certain pathway bifurcations. A series of previous publications has shown the ability of probabilistic tractography to improve the extent of the bundles over DTI-based tractography while highlighting microstructural changes induced by the tumor (44-46). However, usually direction samples are not drawn out of the whole FOD but from the segment defined by an angular deviation threshold (47). This introduces a limitation to probabilistic methods that is using higher angular deviation thresholds helps in reconstructing more complete bundles while also increasing the false-positive rate (32).

Against this background, we evaluate a novel method for improved fiber tractography of the CST in patients suffering from motor-eloquent HGGs, which aims to specifically tackle the issue of missing fiber branching of currently existing tractography procedures. We evaluate the recently developed multi-level fiber tracking (MLFT) approach that adds branches to the pathways that have been previously reconstructed, but do not reach a predefined target region $(48,49)$. Specifically, we hypothesize that the MLFT algorithm is capable of improving the reconstruction of the CST in the vicinity of a brain tumor when compared to conventionally used DTI-based tractography as well as tractography using CSD.

\section{METHODS}

\section{Study Design and Patient Inclusion}

This study was approved by the local institutional review board and was conducted in accordance with the Declaration of Helsinki. The requirement for written informed consent was waived due to the study's retrospective design.

Patients who underwent brain MRI using a multi-sequence imaging protocol for brain tumors according to clinical indication were retrospectively identified in the institutional Picture Archiving and Communication System (PACS). The time interval for PACS search ranged from February 2019 to February 2020 considering the time point of MRI acquisition. Inclusion criteria were 1) age above 18 years, 2) availability of preoperative 3-Tesla MRI including diffusion-weighted sequences, 3 ) diagnosis of a HGG (based on imaging findings and later confirmation by histopathological evaluation of biopsy probes or tumor tissue harvested during surgical resection), and 4) suspected motor-eloquent tumor location according to preoperative MRI (imaging suggesting infiltration or compression of anatomically suspected cortical motor-eloquent areas and/or suspected close proximity to the CST). The exclusion criteria were 1) artifacts due to implants or motion artifacts in imaging data according to visual image evaluation (e.g., non-diagnostic image quality due to patient movement during image acquisition), and 2) previous brain surgery.

Overall, 40 patients fulfilled the inclusion criteria and were considered for this study. Clinical details including demographics and final histopathological tumor grading were extracted from electronic health records of these patients.

\section{Magnetic Resonance Imaging}

Cranial MRI was performed in the preoperative routine setting. All imaging considered in this study was acquired on the same two 3Tesla scanners (Achieva dStream or Ingenia; Philips Healthcare, Best, Netherlands) using a 32-channel head coil.

The standardized multi-sequence imaging protocol for brain tumors included a three-dimensional (3D) fluid attenuated inversion recovery (FLAIR) sequence (repetition time [TR]/ echo time [TE]: $4800 / 277 \mathrm{~ms}, 1 \mathrm{~mm}^{3}$ isovoxel covering the whole head), an axial T2-weighted sequence (TR/TE: 3396/87 $\mathrm{ms}$, voxel size of $0.36 \times 0.36 \times 4 \mathrm{~mm}^{3}$ ), a diffusion-weighted sequence (TR/TE: $5000 / 78 \mathrm{~ms}$, voxel size of $2 \times 2 \times 2 \mathrm{~mm}^{3}$, one volume at $\mathrm{b}=0 \mathrm{~s} / \mathrm{mm}^{2}, 32$ volumes at $\left.\mathrm{b}=1000 \mathrm{~s} / \mathrm{mm}^{2}\right)$, and a 3D T1-weighted turbo field echo (TFE) sequence (TR/TE: 9/4 ms, 1 $\mathrm{mm}^{3}$ isovoxel covering the whole head) without and with application of a contrast agent using a dose of $0.2 \mathrm{ml}$ per $\mathrm{kg}$ body weight of gadoteric acid (Dotagraf $0.5 \mathrm{mmol} / \mathrm{ml}$; Jenapharm GmbH \& Co. KG, Jena, Germany). Further sequences not related to this study's analyses were acquired by default and used for radiological reporting and imagebased diagnostics.

\section{Data Processing Co-Registration and Segmentation}

First, to avoid errors in the automated structural parcellation due to the presence of pathology and related anatomical distortion, lesion filling for the T1-weighted images was done prior to structural parcellation, which substitutes the tumor volume in the image with data mimicking signal from the healthy tissue (either using noise or healthy tissue simulation). For robust parcellation, in this work we used automated Virtual Brain Grafting (VBG), which enables the generation of a virtual lesion-free T1-weighted image and structural parcellation using FreeSurfer recon-all (https:/github.com/KULRadneuron/KUL_VBG/; KU Leuven, Department of Imaging and Pathology, Translational MRI, Leuven, Belgium) (50, 51). Lesion segmentation required for VBG was obtained fusing eight segmentation algorithms using majority voting from the Brain Tumor Segmentation (BraTS) toolkit $(52,53)$. The BraTS toolkit relies on a multi-modal input (non-contrast and contrast-enhanced T1-weighted images, FLAIR images, and T2-weighted images) and produces segmentation masks that enclose the tumor core (necrotic center and contrast-enhancing tumor parts) and FLAIRhyperintense zones (edema/tumor infiltration), which were further used to compute the respective volumes (by accumulating volumes of each voxel in the respective masks) $(52,53)$. Before performing segmentations, all MRI data were transferred to 
Montreal Neurological Institute (MNI) space (with an isotropic voxel size of $1 \mathrm{~mm}^{3}$ ).

The diffusion-weighted MRI data of the individual patients were corrected for motion and eddy currents, and co-registered to the corresponding T1-weighted images using ExploreDTI (version 4.8.6; http://www.exploredti.com/; PROVIDI Lab, Utrecht, Netherlands) (54). The FODs were estimated using recursive calibration of the response function (55). We used a spherical harmonics order of $L_{\max }=6$. Motor cortex masks were assembled from precentral, postcentral, and paracentral lobule segmentations (Figure 1A) obtained with FreeSurfer (version 6.0.0; http://surfer.nmr.mgh.harvard.edu; Laboratory for Computational Neuroimaging, Charlestown, MA, USA) (51) using the Desikan-Killiany atlas (56). All image co-registrations and segmentations were visually inspected for quality and, in case of segmentations, manually corrected by a neuroradiologist when necessary.

\section{Fiber Tacking Algorithms}

Three deterministic tractography approaches were used in this study to reconstruct the CST of both hemispheres: DTI-based tractography, CSD-based tractography, and MLFT. DTI-based tractography was chosen since it is widely used in current clinical practice $(36,57)$. This algorithm propagates fiber streamlines into the main direction of the estimated diffusion tensor. However, it leads to issues with reconstructing pathways in certain complex situations (e.g., crossing or kissing fibers) when the estimated diffusion becomes closer to isotropic and the main tensor direction may not coincide with any of the underlying fiber orientations $(38,39)$. CSD-based tractography has improved the specificity compared to DTI-based tractography given higher angular resolution and an ability to disentangle more complex fiber configurations $(41,42)$. During pathway propagation each time the algorithm chooses an FOD peak that minimizes angular deviation from the previous step. The CSD approach was included as it has shown to be capable of adequately accounting for crossing fiber configurations, and it serves as a basis for the MLFT algorithm.

The recently proposed MLFT algorithm reconstructs bundles as multi-level structures, with the exact number of levels defined by the user $(48,49)$. Given a seed and a target region, MLFT aims to iteratively improve bundle reconstruction by adding pathways with high angulation reaching the target region $(48,49)$. At each iteration, MLFT propagates pathways from a set of seed points using deterministic CSD-based tractography that takes every step into the direction of a FOD peak the least deviating from the direction of the previous step. After propagation, the points of the pathways that did not reach the target region are used as seed points for the following iteration. Their initial directions are then defined as the peaks of the corresponding FODs that were ignored during propagation. If a seed point corresponds to multiple unused FOD peaks, it is duplicated to allow propagating each of the alternative directions. The rest of the pathways reaching the target region are forming a new level of the reconstruction. They are concatenated with the segment of the pathway they branched from that originates from the prior seed point set. This procedure is repeated for a predefined number of iterations. For the CST reconstruction, two levels (iterations) were used in this study. Thus, by extending the reconstruction with each new level, MLFT is attempting to account for branching fibers. It was also shown to preserve topography of the bundles $(48,49)$.

Additionally, it can be noticed that the reconstructions performed with deterministic CSD-based tractography are essentially the first level of the MLFT reconstructions. Thus, the extent of MLFT reconstructions will always at least cover that of the CSD-based algorithm.

\section{Tractography Setup}

To reconstruct the CST within each hemisphere, the seed region was placed in the single-slice transverse cross-section of the pontine level of the brain stem as obtained from brain parcellations (Figure 1B) (58). Motor cortex masks assembled from the segmentation of precentral, postcentral, and paracentral gyri of the left and right hemisphere, respectively, were set as target regions for MLFT and as a region of interest (ROI) to filter the results of DTI- and CSD-based tractography (Figure 1A). Five seed points were sampled per voxel in the seed mask at a single-slice level in the superior part of the brain stem, ensuring that all the points were on the same transverse plane. The tractography step was set to half a voxel size, the angular threshold was set to $45^{\circ}(48,49)$. For MLFT and CSD-based tractography the FOD peak threshold amplitude was set to 0.08 , which was chosen empirically based on visual inspection of the results and with the aim to increase the volume of the reconstruction without introducing obvious false-positive

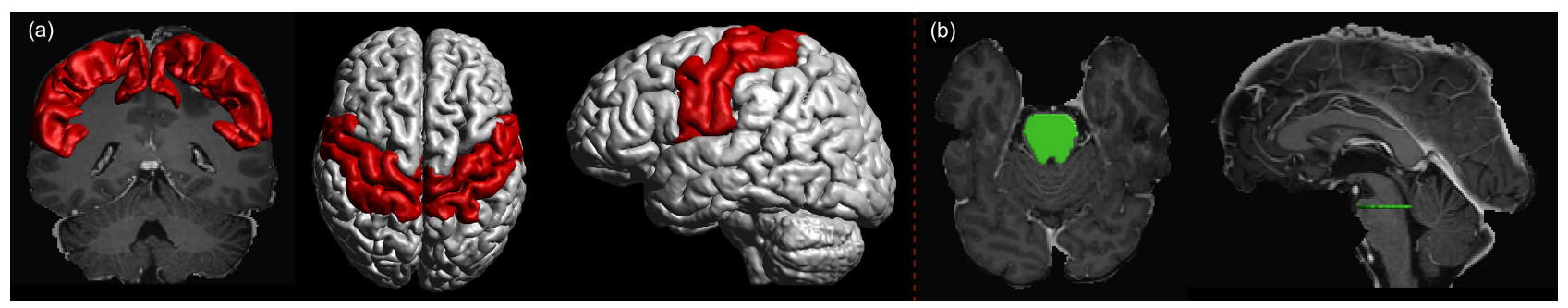

FIGURE 1 | (A) Motor cortex mask (red) was assembled using precentral, postcentral, and paracentral gyri as segmented using FreeSurfer. The motor cortex mask was used as a target region. (B) The seed region (green) was defined as a cross-section of the brain stem at the pontine level. 
pathways. For DTI-based tractography the fractional anisotropy (FA) threshold was set to 0.1 . The number of iterations was set to 2 for MLFT. Additionally, due to reconstruction of CST branches for the left and right hemisphere from the same seed region in the brain stem, interhemispheric connections were filtered out. Additional experiments evaluating algorithms on fine-grained target regions are provided in the Supplementary Material.

\section{Fiber Tracking Evaluation Qualitative Assessment}

Visual image evaluation was performed by a neuroradiologist (7 years of experience in neuroradiological imaging) using ExploreDTI. The reconstructed bundles were rendered in the same scene as the contrast-enhanced T1-weighted images for an interactive assessment of the course of the CST and its relation to the tumor.

Patients were pseudonymized during all visual image evaluations. In detail, datasets stemming from DTI-based tractography, CSD-based tractography, and MLFT were opened during three rounds of evaluation, with each round randomly including one of those tractography results per patient. Between each round of assessment, an interval of at least two weeks was established to minimize recall bias. Both the tumor-affected and unaffected hemispheres were separately evaluated per patient. First, the course of the reconstructed CST through anatomical landmarks known to be key for the descending CST (ipsilateral internal capsule and cerebral crus at the level of the brain stem) was assessed in binary fashion (CST passing through/not passing through internal capsule and cerebral crus). Second, for tractography within the tumor-affected hemisphere, the neuroradiologist assessed whether the reconstructed CST appeared to be unaffected (no contact and no visually identifiable deviation), spatially deviated, infiltrated or split, or destroyed (entire or partial disintegration of the CST) due to the tumor mass, similar to previous work on qualitative evaluation of fiber tract anatomy (59).

\section{Quantitative Assessment}

Quantitative assessment of the CST bundles reconstructed with DTI-based, CSD-based tractography, and MLFT was performed, including radial extent and coverage of reconstructed fibers. The radial extent (in ${ }^{\circ}$ ) of the CST was calculated to show how much of the motor cortex is covered, which was achieved by computing the size of the segment of the coronal motor mask projection covered by the CST. Thus, the motor mask projection defines an $\operatorname{arc}$ of $90^{\circ}$, and the overlap of the bundle visitation mask on the motor cortex defines segments on the arc that constitute the radial extent. The difference in radial extents of the bundles reconstructed in tumoraffected and unaffected hemispheres was compared. Outliers in the difference distribution were detected as patients falling into the distribution tails and accounting for about $5 \%$ of the distribution. The threshold for the detection was calculated using the $2 \sigma$ rule, where $\sigma$ is the standard deviation (SD).

Given its wide use in clinical routine, DTI-based tractography was considered a baseline of comparison for the assessment of the algorithms regarding bundle trajectory. Thus, in order to assess the coherence of the CSD-based tractography and MLFT reconstructions with the DTI-based tractography results, coverage of the bundles generated with DTI-based tractography by the ones generated with CSD and MLFT was calculated. For calculation, binary visitation masks were created of the reconstructed bundles (with voxels being set to 1 if at least one pathway passed through it). Then, the part of the DTIreconstructed bundle's mask intersecting with corresponding masks of the MLFT and the CSD-based reconstructions was calculated (in \%, where 90\% DTI coverage by MLFT would mean that $90 \%$ of the CST volume reconstructed by DTI-based tractography is also included in the respective reconstructed bundle when MLFT is used as the tractography algorithm in the same patient). The masks consisted of the voxels visited by the corresponding bundle (voxel contains at least one pathway point). All computations for quantitative image assessment were performed using in-house developed MATLAB scripts (version R2018b; The MathWorks Inc., Natick, MA, USA).

\section{Statistics}

For statistical data analyses, SPSS (version 26.0; IBM SPSS Statistics for Windows, IBM Corp., Armonk, NY, USA) and SciPy library [version 1.3.1; https://www.scipy.org/scipylib/ (60)] were used. In all statistical tests a significance level of $\alpha=0.05$ was used.

Descriptive statistics included mean $\pm \mathrm{SD}$, ranges, and absolute or relative frequencies. For qualitative image assessment in the tumor-affected hemisphere, Chi-squared tests were conducted to test for differences in the spatial characteristics of the CST (unaffected, spatially deviated, infiltrated/split, or destroyed) between DTI-based tractography, CSD-based tractography, and MLFT. For quantitative image assessment, the tractography algorithms were first compared to each other based on the radial extents of the reconstructions, separately for the unaffected and tumor-affected hemispheres and for the right versus left hemispheres, using two-sided Wilcoxon signed-rank tests. Furthermore, Wilcoxon signed-rank tests were used to compare the radial extents achieved by the same algorithm in affected and unaffected hemispheres, respectively.

Additionally, the coverage of the DTI-based reconstruction of the CST by CSD-based tractography and MLFT was compared for unaffected and tumor-affected hemispheres using two-sided Wilcoxon signed-rank tests. This allowed to assess if the presence of the tumor and related mass effects caused a significant change in the results of MLFT and CSD-based tractography compared to DTI-based tractography for tract coverage. In addition, correlations between the ratio of DTI-based reconstructions of the CST covered by CSD-based tractography or MLFT with the tumor core volumes or FLAIR-hyperintensity zone volumes were calculated using Pearson correlation coefficients.

\section{RESULTS}

\section{Patient Cohort}

Forty patients (mean age: $62.6 \pm 13.4$ years, age range: 29.5 - 85.9 years, 17 females and 23 males) with a diagnosis of HGG and suspected motor-eloquent tumor location were included. Table 1 provides further cohort details. 
TABLE 1 | Characteristics of the study cohort.

\begin{tabular}{|c|c|c|}
\hline \multicolumn{2}{|l|}{ Item } & $\begin{array}{c}\text { Value } \\
62.6 \pm 13.4\end{array}$ \\
\hline (years; mean \pm SD \& range) & & $(29.5-85.9)$ \\
\hline (\% of patients) & Female & 42.5 \\
\hline Affected hemisphere & Left & 40.0 \\
\hline (\% of patients) & Right & 60.0 \\
\hline Surgical procedure & Biopsy & 22.5 \\
\hline Extent of resection & STR & 25.8 \\
\hline (\% of patients) & GTR & 74.2 \\
\hline Tumor grade & WHO grade III & 12.5 \\
\hline (\% of patients) & WHO grade IV & 87.5 \\
\hline $\begin{array}{l}\text { Tumor core volume } \\
\left(\mathrm{mm}^{3}, \text { mean } \pm \text { SD \& range }\right)\end{array}$ & & $\begin{array}{c}47,997.4 \pm 39,098.9 \\
(2,582-170,576)\end{array}$ \\
\hline
\end{tabular}

SD, standard deviation; WHO, World Health Organization; STR, subtotal resection; GTR, gross total resection; RTX, radiotherapy.

\section{Qualitative Assessment}

Representative exemplary cases for CST reconstruction by DTIbased tractography, CSD-based tractography, and MLFT are shown in Figures 2, 3. The reconstructed CST passed through the internal capsule and cerebral crus as key anatomical landmarks for both hemispheres of all enrolled patients.

The reconstructed CST bundle was unaffected by or not in contact with the tumor mass in $n=15,8$, and 4 patients for DTIbased tractography, CSD-based tractography, and MLFT, respectively, and did not fulfill the criteria of a disintegrated course in any of the patients. Furthermore, the reconstructed CST bundle was deviated in $\mathrm{n}=24,26$, and 22 patients, respectively. It appeared to be infiltrated/split in $n=1,6$, and 14 patients when using DTI-based tractography, CSD-based tractography, or MLFT, respectively. There was a statistically significant difference in these spatial characteristics of the CST depending on the tractography approach chosen $(\mathrm{p}=0.0006)$.

\section{Quantitative Assessment Radial Extent}

The radial extents of the CST branches reconstructed with the three tractography algorithms are presented in Figures 4, 5. The MLFT algorithm consistently provides increased radial extent when compared to both CSD-based and DTI-based tractography in all patients. In addition, when comparing radial extents between reconstructions from the three different tractography algorithms, results were statistically significant throughout (DTI vs. CSD/DTI vs. MLFT/CSD vs. MLFT: $\mathrm{p}<$ 0.05 each for tumor-affected vs. unaffected as well as right vs. left hemispheres; Table 2).

Each of the algorithms produced CST reconstructions with higher median radial extent of the reconstructions for the tumoraffected hemispheres as compared to the unaffected hemispheres, with a statistically significant difference only for MLFT (median radial extent for tumor-affected vs. unaffected hemisphere - DTI: $19.46^{\circ}$ vs. $18.99^{\circ}, \mathrm{p}=0.8931$; CSD: $30.54^{\circ}$ vs. $27.63^{\circ}, \mathrm{p}=0.0546$; MLFT: $81.17^{\circ}$ vs. $\left.74.59^{\circ}, \mathrm{p}=0.0134\right)$.
Furthermore, the differences in radial extents of the CST bundles in tumor-affected and unaffected hemispheres were compared (Figure 6). Using $2 \sigma$, three outliers were identified (patients \#8, \#10, and \#37), who were all characterized by extensive mass effect that caused deformation of the CST bundle within the tumor-affected hemisphere, and to a lesser extent also a deviation of the CST within the unaffected hemisphere (Figure 7). Midline shifts can be observed in these three outliers.

\section{Coverage of DTI}

The results on comparing the coverage of DTI-based reconstructions by the corresponding CSD-based and MLFTbased reconstructions are depicted in Figure 8. MLFT provides a higher fraction of coverage of the DTI reconstruction results of the CST when compared to CSD.

Both CSD-based and MLFT results more clearly coincide with DTI-based reconstructions in case of the unaffected hemispheres (median coverage for affected vs. unaffected hemisphere - CSD: $68.16 \%$ vs. $77.59 \%, \mathrm{p}=0.0075$; MLFT: $93.09 \%$ vs. $95.49 \%$; $\mathrm{p}=$ $0.0046)$, while the reconstructions in the tumor-affected hemisphere are characterized by a higher mismatch. Yet, for the MLFT median coverage is $>90 \%$ for both the unaffected and tumor-affected hemisphere, which contrasts with the CSD-based reconstruction with a median coverage of $<80 \%$. The two patients that had the lowest DTI coverage (lower than $75 \%$ ) in the tumor-affected hemisphere by the MLFT reconstructions were subjects with extensive mass effects and high tumor volume, namely patients \#5 and \#17. The lowest DTI coverage achieved with CSD-based tractography is also attributed to patient \#5. For these patients, tumor volumes as well as volumes of the FLAIRhyperintense zones ranged in the upper quartile.

The correlation coefficients of the DTI coverage by CSDbased tractography or MLFT with the tumor core (necrotic center and contrast-enhancing tumor parts) and FLAIRhyperintense zones are shown in Table 3. There were statistically significant negative correlations between the 


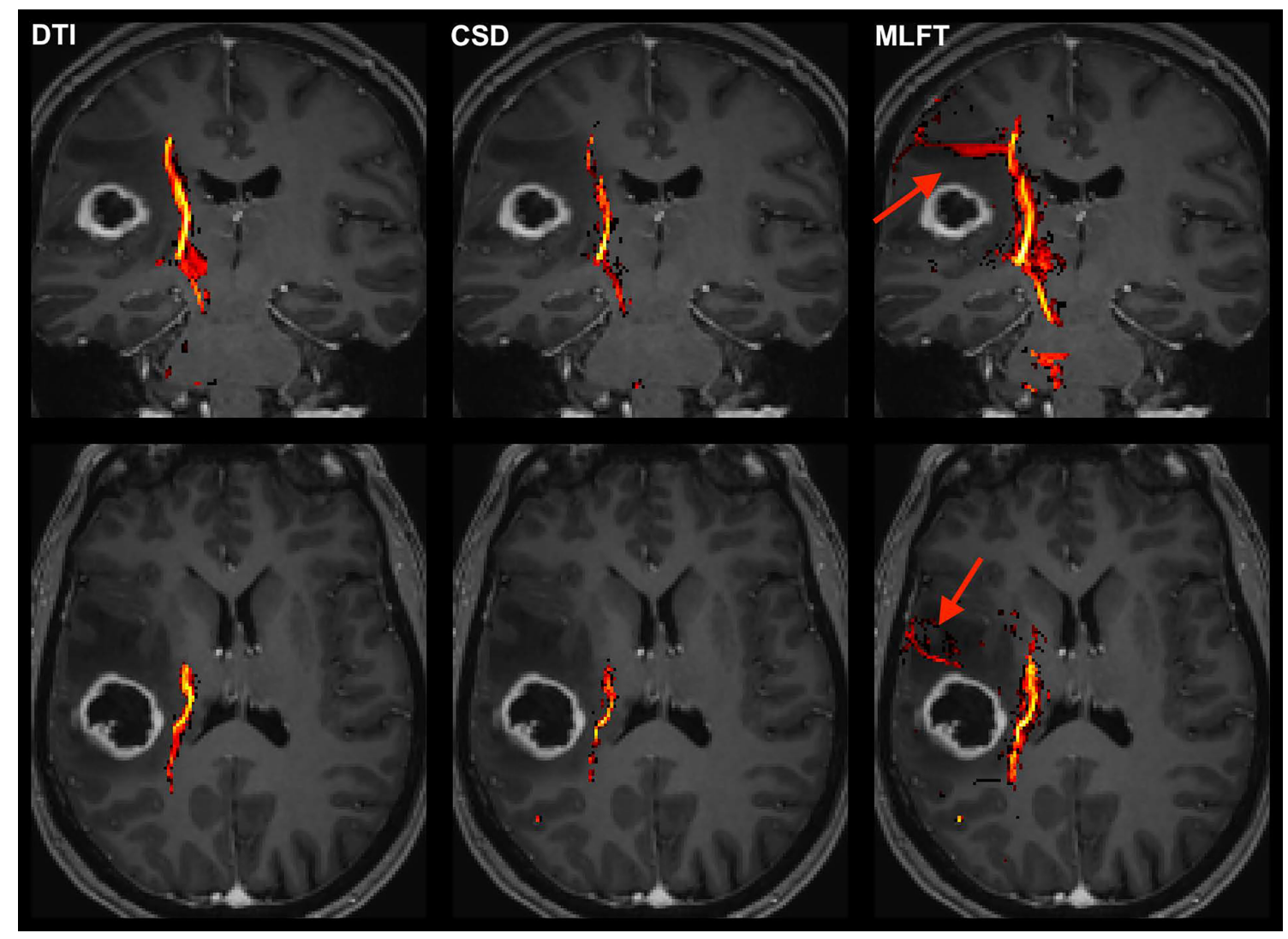

FIGURE 2 | Exemplary case for reconstruction of the corticospinal tract (CST) depending on the algorithm used for tractography. This illustrative exemplary case of a patient suffering from a right-sided high-grade glioma (HGG) shows the reconstructed CST within the tumor-affected hemisphere as derived from diffusion tensor imaging (DTI)-based tractography, constrained spherical deconvolution (CSD)-based tractography, and multi-level fiber tracking (MLFT). The CST reconstructions are fused with axial and coronal contrast-enhanced T1-weighted images to outline the lesion-to-CST relationship as well as the CST volume and course. The MLFT approach enables fiber tracking with a larger radial extent, thus displaying also fanning of the CST and fibers with acute angles (red arrow).

volume of the FLAIR-hyperintense zone and the DTI coverage (CSD: $\mathrm{r}=-0.52, \mathrm{p}=0.0006$; MLFT: $\mathrm{r}=-0.52, \mathrm{p}=0.0005$ ). Likewise, there were statistically significant negative correlations between the volume of the tumor core plus the volume of the FLAIR-hyperintense zone and DTI coverage (CSD: $\mathrm{r}=-0.48, \mathrm{p}=$ 0.0018; MLFT: $\mathrm{r}=-0.47, \mathrm{p}=0.0021$ ).

\section{DISCUSSION}

In this work we evaluated a novel tractography algorithm, MLFT, to achieve improved reconstruction of the CST in patients with motor-eloquent HGG. The MLFT algorithm was compared to deterministic DTI-based and deterministic CSD-based tractography of the CST of both hemispheres. The main findings are as follows: 1) the algorithm chosen for tractography had significant impact on the spatial course, volume, and shape of the CST, with MLFT-based reconstructions showing significantly higher radial extents; 2) compared to deterministic CSD-based tractography, MLFT showed higher coincidence with the DTIbased reconstructions, with a median coverage of $>90 \%$ for both the tumor-affected as well as unaffected hemispheres; and 3) coverage of the CST as tracked by the DTI-based algorithm was significantly negatively associated with tumor-related mass effects (as estimated by volumes of tumor core and FLAIR-hyperintense zones) for both the CSD-based algorithm and MLFT.

Tractography of WM structures such as the CST is frequently employed for preoperative planning and intraoperative resection guidance in patients with intracranial neoplasms, using primarily DTI-based approaches (27-31). However, DTIbased tractography has several known limitations that may hamper value for clinical applications, including its limited ability to resolve geometrically complex situations such as crossing or kissing fibers $(38,39)$. Previous research has shown that using more advanced techniques, such as q-ball and CSDbased fiber tractography, may lead to improved results $(37,61$, 62). In turn, the proposed MLFT algorithm is developed from CSD-based tractography and similarly propagates fiber pathways based on FOD peaks. However, unlike CSD-based tractography, MLFT assumes that FOD peaks represent not only fiber crossings, but also indicate fiber branching or high-angular deviation $(48,49)$. Without prior anatomical knowledge, such 


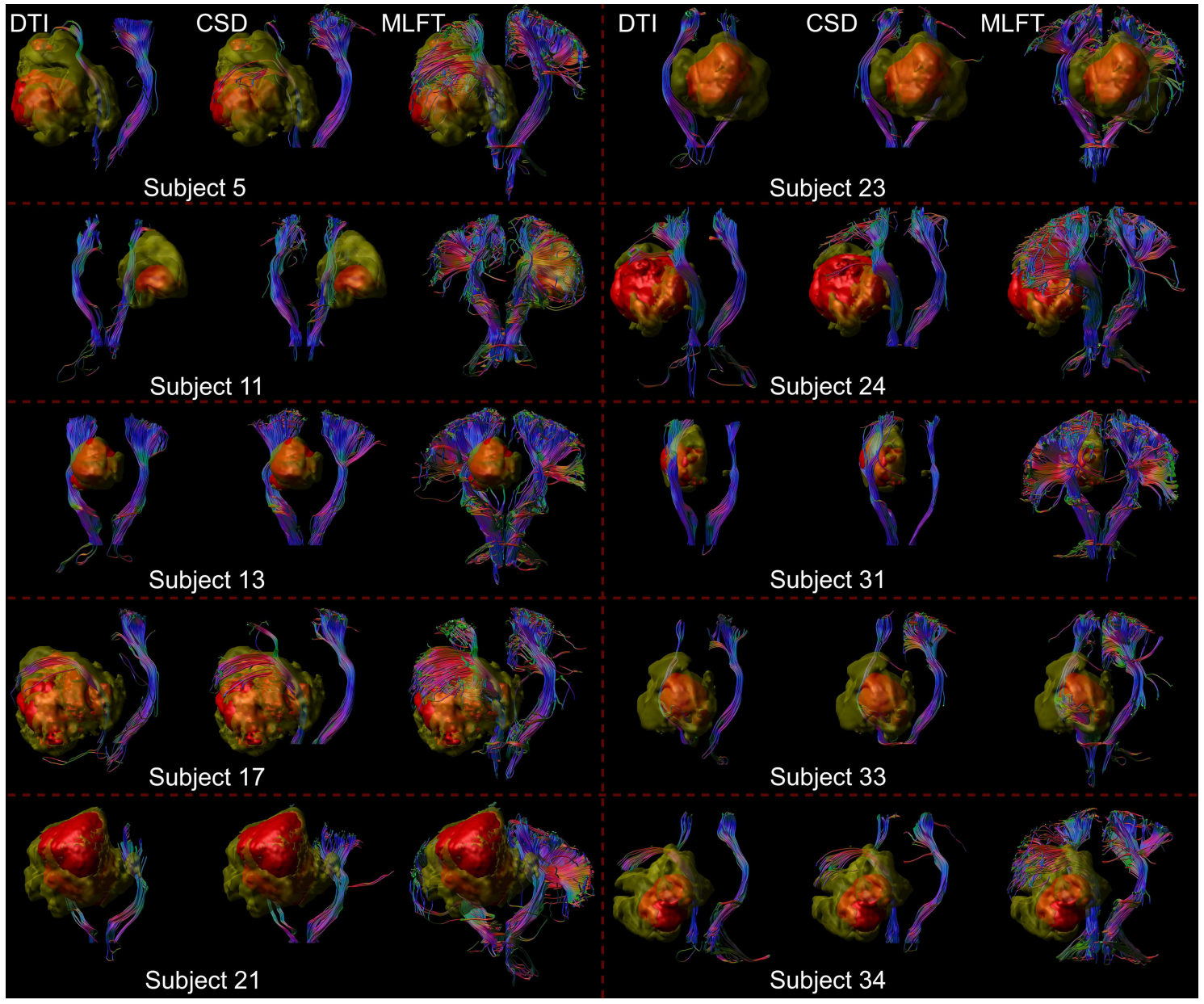

FIGURE 3 | Comparison of reconstructions of the corticospinal tract (CST) depending on the algorithm chosen for tractography. This figure shows reconstructions of the CST within the tumor-affected and unaffected hemispheres in a subset of 10 patients from the cohort, using diffusion tensor imaging (DTI)-based tractography, constrained spherical deconvolution (CSD)-based tractography, and multi-level fiber tracking (MLFI). The tumor core is shown as a red volume, the hyperintense zone in fluid attenuated inversion recovery (FLAIR) sequences is shown as a yellow volume. While CSD-based tractography provides reconstructions comparable to DTI-based tractography, MLFT is able to improve depiction of the extent of the CST fanning of both tumor-affected and unaffected hemispheres.

an approach would be at risk of generating multiple false-positive streamlines, which needs to be avoided particularly for ultimate clinical applicability. In order to prevent a high false-positive rate, MLFT requires well-defined target and seed regions, and if certain pathways do not enter the target area our algorithm checks if any deviation at the previous points would allow reaching the target region. Hence, using the algorithm gives some control over specificity while improving sensitivity.

Reconstructions of the CST using MLFT consistently showed the highest radial extent when compared to DTI- or CSD-based tractography (Figure 3). On average, CSD-based tractography achieved higher radial extent than the DTI-based algorithm (Table 2), while in some individual cases DTI-based tractography outperforms the CSD algorithm for this metric (Figure 4). Yet, MLFT provided CST branches with higher radial extents even for the tumor-affected hemispheres, which may indicate that a more complete reconstruction of particularly highly angulated parts of the CST close to its origin becomes possible when using MLFT (Figures 4, 5). Indeed, based on simulations and preliminary in-vivo imaging in a cohort of healthy subjects, it has been suggested that the fanning close to the motor cortex can be well delineated with $\operatorname{MLFT}(48,49)$. Considering the potential value of a broader fanning and reconstruction of laterally coursing fiber pathways, MLFTderived reconstructions may be of merit since especially marginal fibers can be at risk for damage when aiming at a maximized EOR during surgery of motor-eloquent HGGs.

When comparing radial extents of tumor-affected and unaffected hemispheres, the observed differences are mostly comparable across the algorithms (Figure 6). Further, we separately explored the outliers with values above the mean + $2 \sigma$, given that they were of most interest, while any cases below the mean $-2 \sigma$ were considered in the range of an expected result. As the unaffected hemisphere apparently does not show 

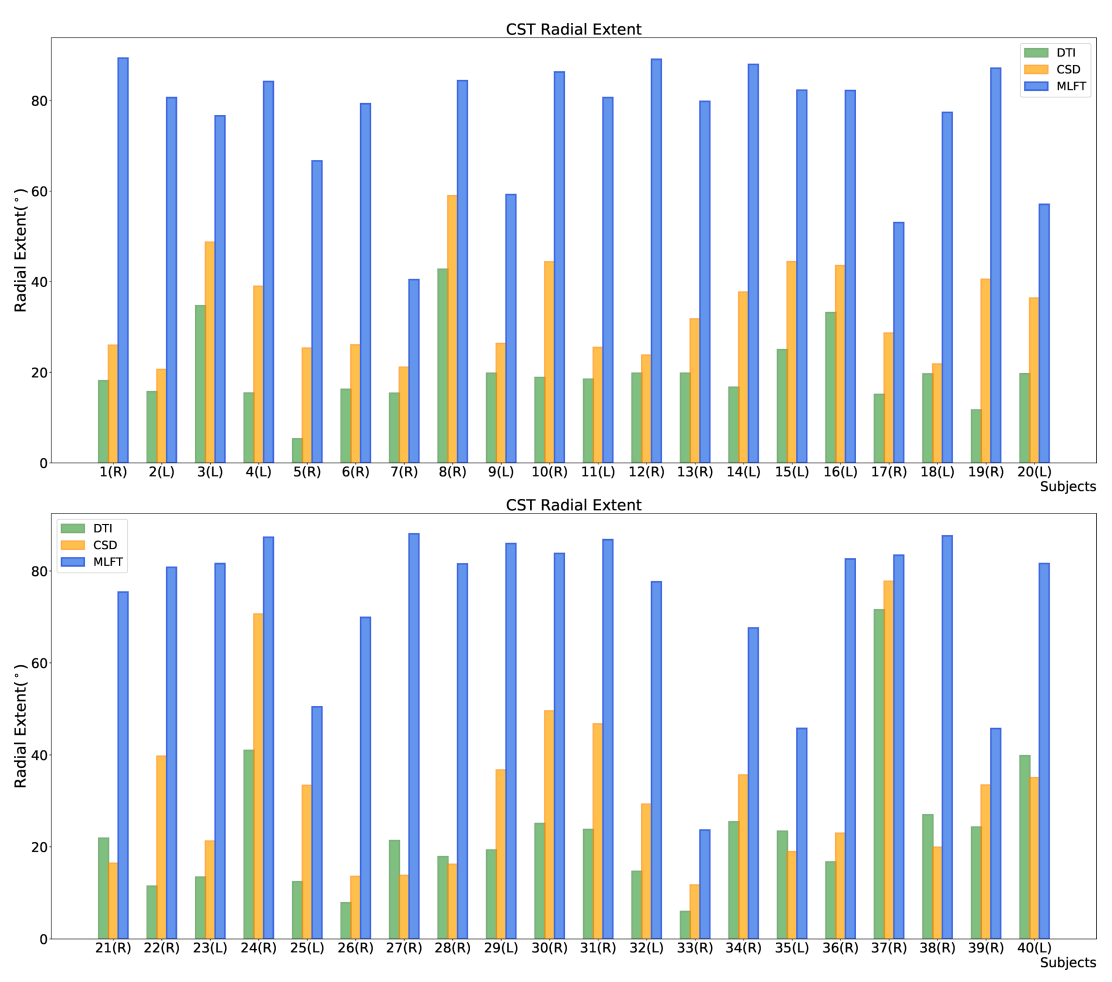

FIGURE 4 | Comparison of the radial extent of the corticospinal tract (CST) branches of the tumor-affected hemispheres. This figure illustrates the radial extent for CST reconstruction using diffusion tensor imaging (DTI)-based tractography (green), constrained spherical deconvolution (CSD)-based tractography (orange), and multi-level fiber tracking (MLFT; blue). The hemisphere affected by the tumor per patient is indicated next to the subject index ( $L-$ left, $R-$ right). Using MLFT led to CST reconstructions with larger radial extent in all patients.

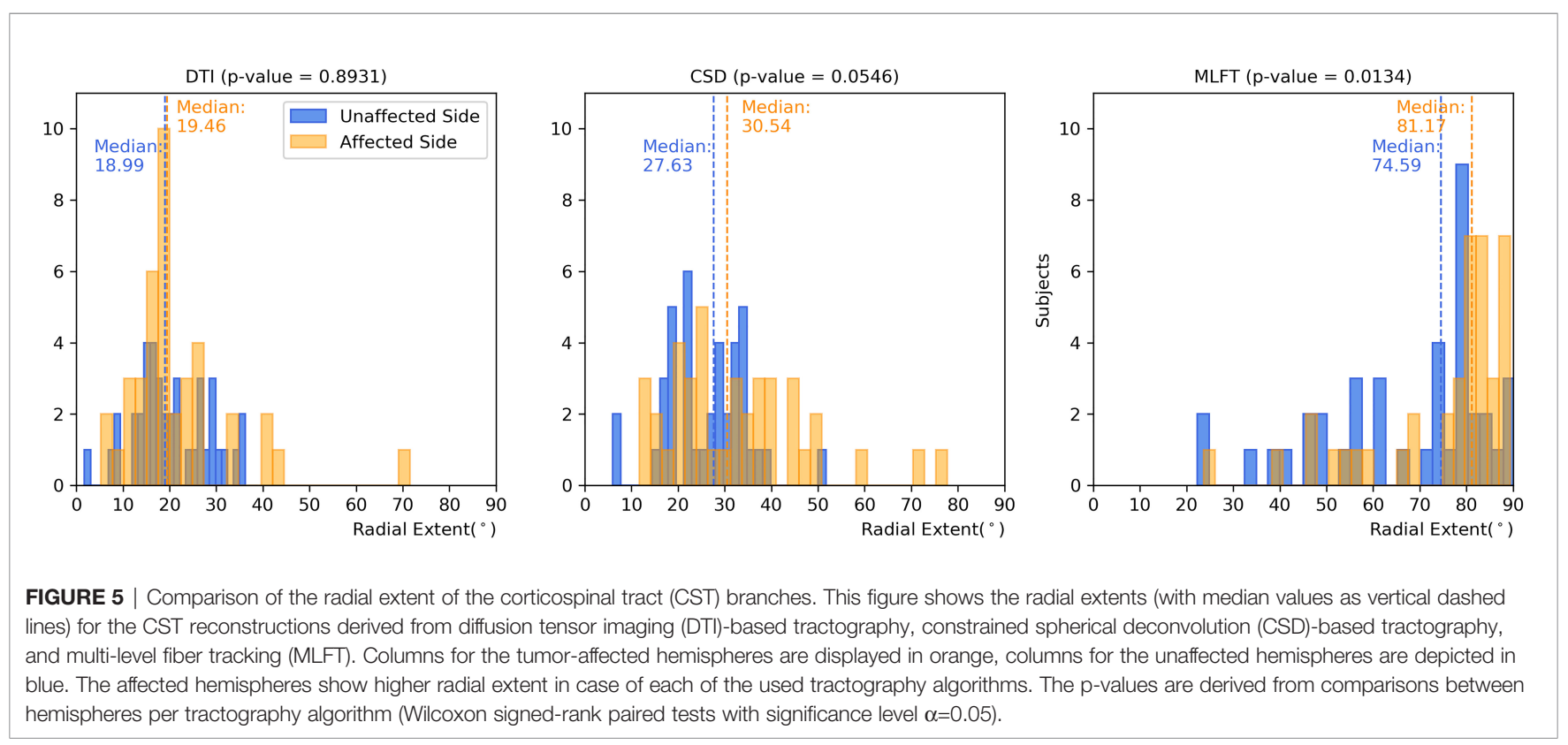

the same changes in microstructure related to a tumor, the CST extent there should be at least comparable. Patients with considerable radial extent differences (above the $2 \sigma$ threshold) are all characterized by mass effects extensive enough to cause midline shift and introduce deformations to the bundle in unaffected hemispheres (Figure 7), while the opposite is not always true. Depending on the distinct location of the tumor, midline shift may not lead to higher radial extent of the CST in 
TABLE 2 | Radial extent of fiber reconstructions.

\begin{tabular}{|c|c|c|c|c|c|c|c|c|c|}
\hline Radial Extent Hemisphere & \multicolumn{3}{|c|}{ Mean $\pm S D,\left(^{\circ}\right)$} & \multicolumn{3}{|c|}{ Range, $\left({ }^{\circ}\right)$} & \multicolumn{3}{|c|}{ P-value } \\
\hline Right & $21.9 \pm 11.7$ & $30.0 \pm 14.6$ & $73.8 \pm 16.1$ & $5.28-71.53$ & $11.68-77.75$ & $23.65-90.45$ & $5.5^{\star} 10^{-5}$ & $3.6^{\star} 10^{-8}$ & $3.6^{*} 10^{-8}$ \\
\hline Affected & $21.6 \pm 11.7$ & $32.8 \pm 14.6$ & $74.8 \pm 15.6$ & $5.28-71.53$ & $11.68-77.75$ & $23.65-89.41$ & $1.1^{*} 10^{-6}$ & $3.6^{\star} 10^{-8}$ & $3.6^{*} 10^{-8}$ \\
\hline Unaffected & $20.3 \pm 8.0$ & $26.1 \pm 8.9$ & $66.7 \pm 17.9$ & $1.75-36.22$ & $5.97-51.72$ & $22.31-90.45$ & $1.5^{\star} 10^{-5}$ & $3.6^{\star} 10^{-8}$ & $3.6^{*} 10^{-8}$ \\
\hline
\end{tabular}

This table shows the mean $\pm S D$ and ranges for the radial extents of CST reconstructions with the three different algorithms used (DTI-based tractography, CSD-based tractography, and MLFT). Discrimination is made between left and right hemispheres as well as tumor-affected and unaffected hemispheres. P-values were computed for the comparisons of radial extents derived from the different algorithms (Wilcoxon signed-rank paired tests with significance level $\alpha=0.05$ ). CST, corticospinal tract; SD, standard deviation; DTI, diffusion tensor imaging; CSD, constrained spherical deconvolution; MLFT, multi-level fiber tracking

the unaffected hemisphere; for instance, in cases of a more anterior tumor location and midline shift occurrence, no significant alteration was observed in radial extents. Regarding the reasons for the occurred differences, lateral components of the reconstructed CST pathways might be re-oriented as a result of WM compression causing smoothing of the acute angles of the fibers, which allowed the tractography algorithms to reconstruct them. Similarly, the CST pathways in the unaffected hemisphere could have been compressed and deviated in such a way that the angular resolution of the acquisition would not allow to resolve all fiber orientations properly, given that the acquired sequence only included 32 directions by default. In this regard, increasing the order of spherical harmonics used to estimate FODs would also increase the angular resolution, potentially solving the issue (41). Yet, this would require inclusion of a higher number of gradient directions in the sequence (63). At the same time, MLFT already reconstructs pathways closer to the tumor (Figure 2), hinting at potentially small tumor-to-CST distances that may exert impact on neurosurgical planning and have implications for patient outcome in terms of motor function and avoidance of surgery-related functional decline.

While the general trajectory and shape of the DTI-based reconstructions tend to be maintained by both MLFT and CSD- based tractography, MLFT improves the coverage of CST reconstructions as provided by DTI-based tractography over those taken from the CSD algorithm (Figure 8). Notably, the median coverage of DTI-based CST reconstructions by the CST as delineated with MLFT was higher than $90 \%$ for both tumoraffected and unaffected hemispheres. This indicates that the approach does not considerably increase the false-negative rate, while performing better than the CSD algorithm that provides a median coverage below $80 \%$. At the same time, the coverage of the DTI-based CST reconstruction by MLFT as well as CSDbased tractography is inversely correlated to measures for tumorrelated mass effects (as estimated by volumes of tumor core and FLAIR-hyperintense zones), which might reflect the effect of tumor-induced WM changes on the estimated orientation distribution by either of the used models (Table 3). However, the question arises which method comes closest to the in-vivo course and architecture of the CST. The gold standard to test this would be intraoperative DES, which has not been applied to evaluate CSD or MLFT results because of this study's retrospective design. Yet, there seems high agreement in neurosurgical oncology that techniques should move beyond DTI-based tractography to improve accuracy of tracking results (34-37).

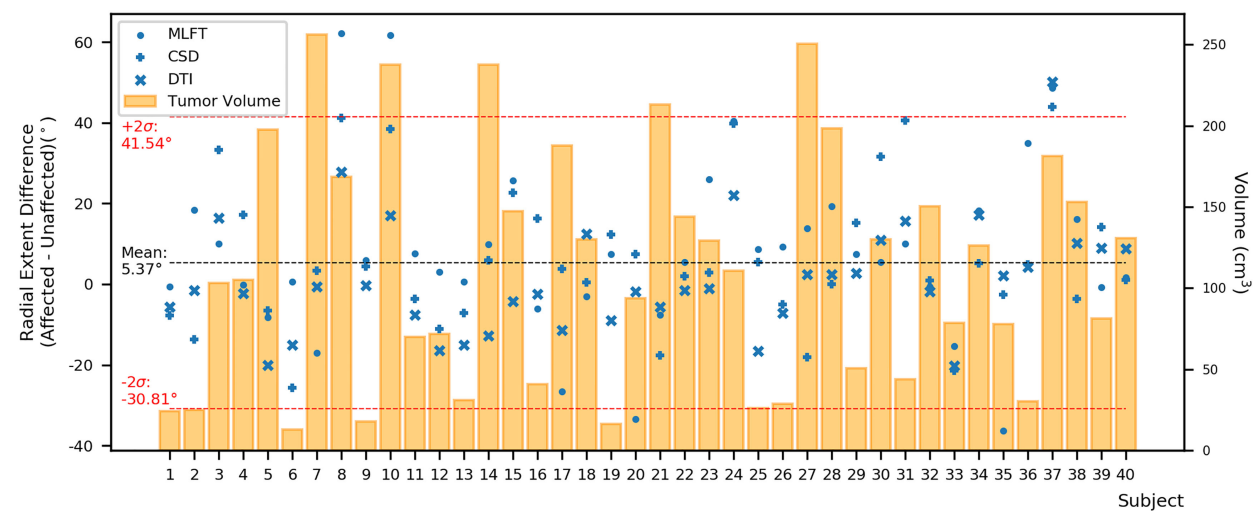

FIGURE 6 | Differences between the radial extent of tumor-affected and unaffected hemispheres. This figure shows the radial extent differences in relation to combined tumor and FLAIR-hyperintense zone volumes (orange) using the mean (black dashed line) with +/- 2 standard deviation (SD, provided as $\sigma$; red dashed lines) to identify potential outliers. Circles represent data points for the corticospinal tract (CST) as derived from multi-level fiber tracking (MLFT), while + represents data points derived from constrained spherical deconvolution (CSD)-based tractography and $\mathrm{x}$ represents data points stemming from diffusion tensor imaging (DTI)based tractography. The outliers with positive radial extent difference are of most interest as they show unexpected behavior with higher radial extent in the tumoraffected hemisphere. 


\begin{tabular}{|c|c|c|c|c|}
\hline & \multicolumn{2}{|c|}{ CSD coverage of DTI } & \multicolumn{2}{|c|}{ MLFT coverage of DTI } \\
\hline & $\mathbf{r}$ & P-value & $\mathbf{r}$ & P-value \\
\hline Tumor Core Volume & -0.25 & 0.12 & -0.24 & 0.14 \\
\hline Volume of FLAIR-hyperintense zone & -0.52 & $<0.01$ & -0.52 & $<0.01$ \\
\hline Volume of Tumor Core + FLAIR-hyperintense zone & -0.48 & $<0.01$ & -0.47 & $<0.01$ \\
\hline
\end{tabular}

This table shows the Pearson correlation coefficient ( $r$ ) and related $p$-values for the correlations between tumor core volume, volume of FLAIR-hyperintense zone, and volume of tumor core plus FLAIR-hyperintense zone and coverage of the DTI-derived CST for reconstructions using CSD-based tractography or MLFT, respectively (significance level $\alpha=0.05$ ). DTI, diffusion tensor imaging; CSD, constrained spherical deconvolution; MLFT, multi-level fiber tracking; FLAIR, fluid attenuated inversion recovery.

One aspect that may further improve tractography using the MLFT algorithm is to combine it with techniques that provide function-based ROIs for seeding. In this regard, previous work has used activation maps derived from functional MRI for ROI placements (64-66). More recently, motor maps derived from navigated transcranial magnetic stimulation (nTMS) have been used for ROI placements (67-72). Of note, it has been demonstrated that nTMS facilitates optimized tracking results for the CST, particularly when the primary motor cortex was in close vicinity of a brain tumor, suggesting that nTMS may be considered the method of choice to achieve proper ROI placements for CST tractography using DTI (71). Comparisons between the three algorithms using nTMS motor maps for seeding may help identify parts of the CST that are underrepresented by DTI- or CSD-based tractography but are evidently connected to the primary motor cortex, which might in particular include fibers with acute angles that could be better delineated with the MLFT method. Additionally, subcortical

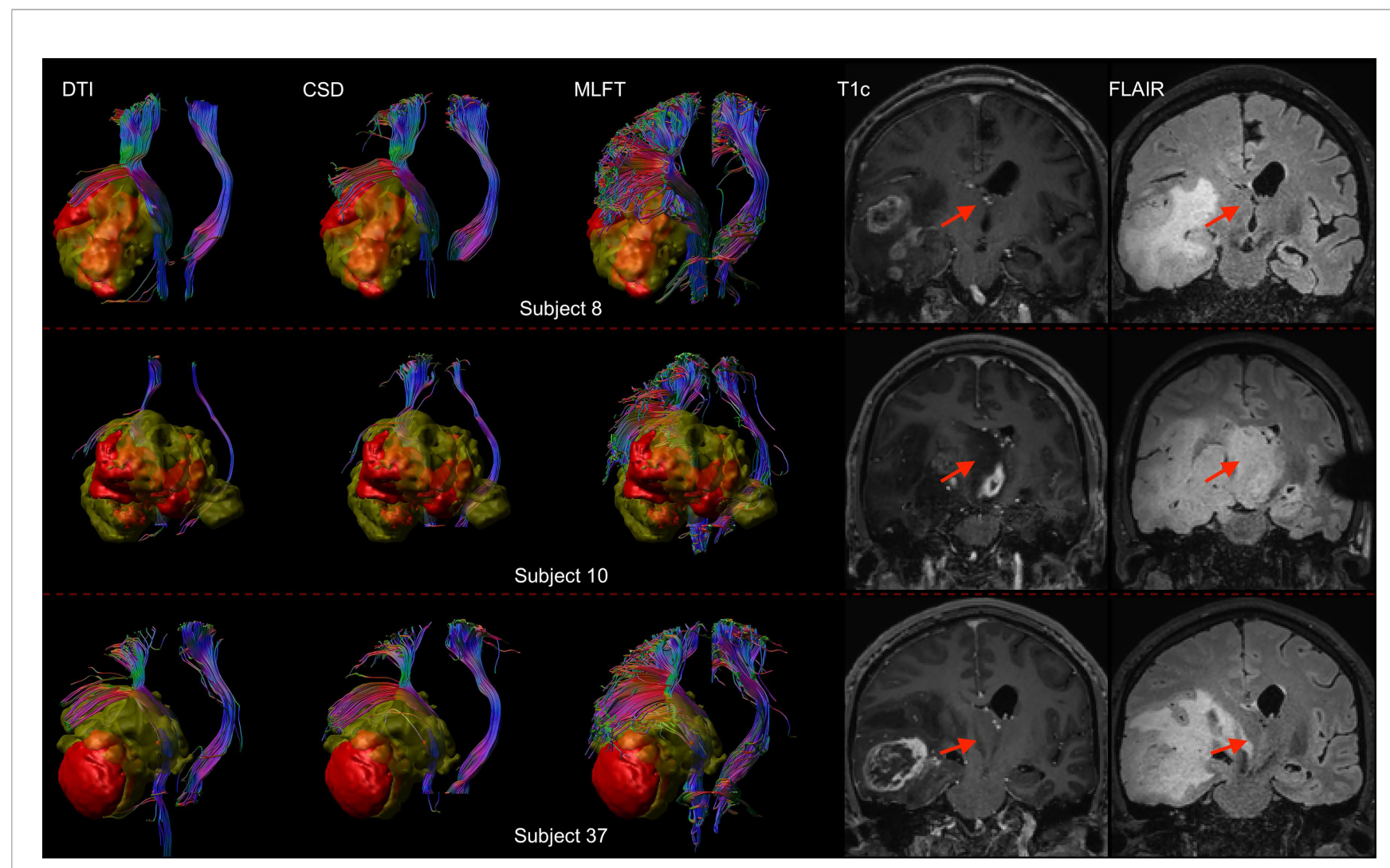

FIGURE 7 | Comparison of reconstructions of the corticospinal tract (CST) depending on the algorithm chosen for tractography in patients with high radial extent in the tumor-affected hemisphere. This figure shows reconstructions of the CST using diffusion tensor imaging (DTI)-based tractography, constrained spherical deconvolution (CSD)-based tractography, and multi-level fiber tracking (MLFT) within the tumor-affected and unaffected hemispheres in the subset of the three patients that were identified as outliers regarding radial extent within affected hemispheres (considering a $2 \sigma$ threshold). The tumor core is shown as a red volume, the hyperintense zone in fluid attenuated inversion recovery (FLAIR) sequences is shown as a yellow volume. These patients were all characterized by extensive mass effect that caused deformation of the CST bundle within the tumor-affected hemisphere as well as, to a lesser extent, within the unaffected hemisphere with considerable midline shift (red arrow in coronal contrast-enhanced T1-weighted and coronal FLAIR images). In all cases, fanning is considerably improved particularly in the tumor-affected hemispheres when using the MLFT algorithm, with only few fibers with acute angles being displayed adjacent to the tumor masses when using DTI-based tractography. 

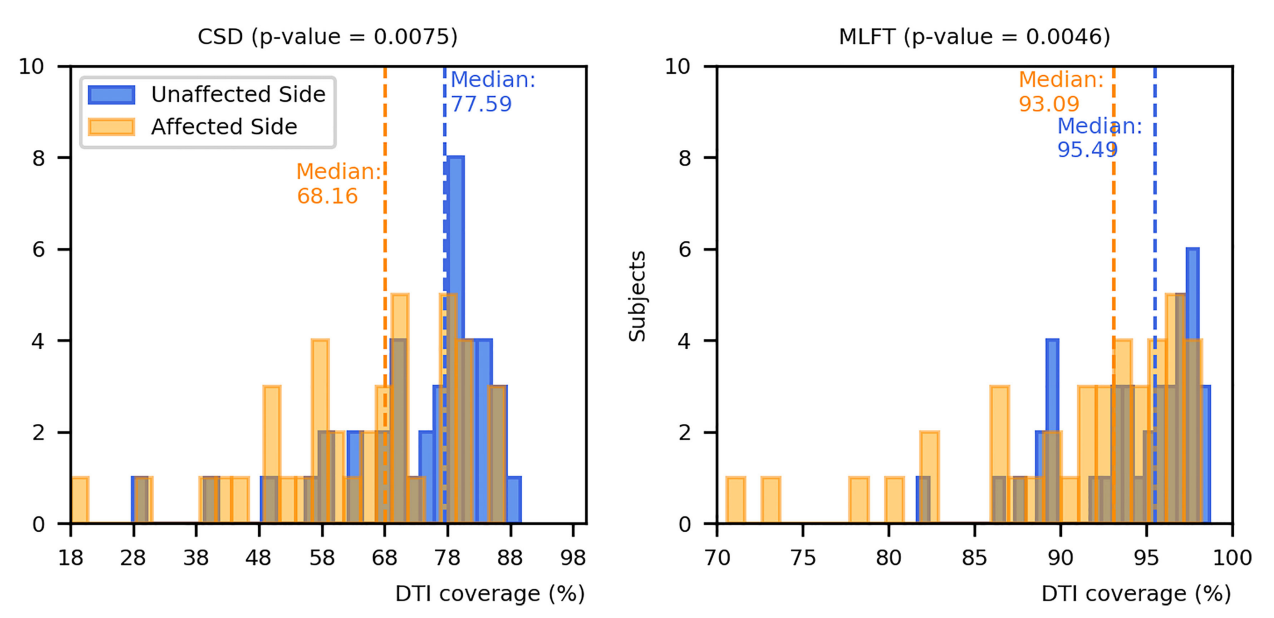

FIGURE 8 | Comparison of coverage for corticospinal tract (CST) branches. This figure shows the coverage (with median values as vertical dashed lines) of the CST reconstructions derived from diffusion tensor imaging (DTI)-based tractography by constrained spherical deconvolution (CSD)-based tractography or multi-level fiber tracking (MLFT). Columns for the tumor-affected hemispheres are displayed in orange, columns for the unaffected hemispheres are depicted in blue. The affected hemispheres show lower coverage compared to the tumor-unaffected hemispheres. The p-values are derived from comparisons between hemispheres per tractography algorithm (Wilcoxon signed-rank paired tests with significance level $\alpha=0.05$ ).

language network analysis using nTMS-defined ROIs could be part of future work, as to date it has predominantly been performed with DTI-based tractography $(68,73-75)$.

The main limitation of the MLFT method is related to the accuracy of the estimated FODs in the WM. In a clinical setting neither the number of acquired directions nor b-values are routinely set high, and the accuracy of the fitted diffusion models may therefore be hampered, as the FODs have to be represented by lower-order spherical harmonics. Additionally, the FOD algorithm used does not estimate separate response functions for different tissues (55). An acquisition with multiple diffusion weightings (e.g., multi-shell imaging) would allow to use FOD estimation algorithms that are capable of differentiating multiple tissues (76). Another important limitation of this study is the absence of a correlation of the tractography results derived from CSD and MLFT to findings of intraoperative DES, as it would allow estimation of the sensitivity and specificity rates of these tractography algorithms. This is due to the study's retrospective character, while conventionally used DTI-based tractography for delineation of the CST has, however, been performed and considered for presurgical planning and intraoperative guidance within the scope of the standard of clinical care.

\section{CONCLUSION}

The results of this work suggest that tractography of the CST in patients harboring motor-eloquent HGGs may be improved using the proposed MLFT method. This advancement of the CSD principle enabled delineation of the CST with significantly increased radial extent for fibers close to the motor cortex, while maintaining coincidence with DTI-reconstructed CST bundles.

\section{DATA AVAILABILITY STATEMENT}

The data analyzed in this study is subject to the following licenses/restrictions: Analyzed dataset is a subject to medical secrecy and cannot be shared. The derivatives may be provided by the authors upon request. Requests to access these datasets should be directed to Andrey Zhylka, a.zhylka@tue.nl.

\section{ETHICS STATEMENT}

The studies involving human participants were reviewed and approved by the Ethikkommission der Technischen Universität München. Written informed consent for participation was not required for this study in accordance with the national legislation and the institutional requirements.

\section{AUTHOR CONTRIBUTIONS}

Conceptualization was performed by AZ, NS, ADL, AL, and JP. Methodology involved AZ, NS, and ADL. Software involved AZ, FK, AR, and AL. Validation was performed by NS. Formal analysis performed by $\mathrm{AZ}$ and NS. Investigation was performed by AZ and NS. Resources provided by JG, BW, BM, SK, CZ, JK, and SS. Data curation was performed by AZ and NS. Writing - original draft preparation was performed by AZ and NS. Writing - review and editing was performed by AZ, NS, FK, AR, ADL, JG, BW, BM, SK, CZ, JK, SS, AL, and JP. Visualization was performed by AZ and NS. Supervision was performed by NS, $\mathrm{BM}, \mathrm{CZ}, \mathrm{JK}, \mathrm{SS}, \mathrm{AL}$, and JP. Project administration was performed by NS, CZ, and JK. All authors contributed to the article and approved the submitted version. 


\section{FUNDING}

AZ is supported by the European Union's Horizon 2020 research and innovation program under the Marie Sklodowska-Curie grant agreement [765148]. BM, BW, and FK are supported through the SFB 824, subproject B12. This project was furthermore supported by the Deutsche Forschungsgemeinschaft (DFG) through TUM International Graduate School of Science and Engineering (IGSSE), GSC 81. The funders were not involved in the study design, collection, analysis, interpretation of data, the writing of this article or the decision to submit it for publication. All authors declare no other competing interests.

\section{REFERENCES}

1. Ostrom QT, Bauchet L, Davis FG, Deltour I, Fisher JL, Langer CE, et al. The Epidemiology of Glioma in Adults: A "State of the Science" Review. NeuroOncology (2014) 16(7):896-913. doi: 10.1093/neuonc/nou087

2. Ostrom QT, Cote DJ, Ascha M, Kruchko C, Barnholtz-Sloan JS. Adult Glioma Incidence and Survival by Race or Ethnicity in the United States From 2000 to 2014. JAMA Oncol (2018) 4(9):1254-62. doi: 10.1001/jamaoncol.2018.1789

3. Wen PY, Kesari S. Malignant Gliomas in Adults. N Engl J Med (2008) 359 (5):492-507. doi: 10.1056/NEJMra0708126

4. Ostrom QT, Cioffi G, Gittleman H, Patil N, Waite K, Kruchko C, et al. CBTRUS Statistical Report: Primary Brain and Other Central Nervous System Tumors Diagnosed in the United States in 2012-2016. Neuro-Oncology (2019) 21(Suppl 5):v1-v100. doi: 10.1093/neuonc/noz150

5. Claes A, Idema AJ, Wesseling P. Diffuse Glioma Growth: A Guerilla War. Acta Neuropathol (2007) 114(5):443-58. doi: 10.1007/s00401-007-0293-7

6. Martinez-Garcia M, Alvarez-Linera J, Carrato C, Ley L, Luque R, Maldonado $\mathrm{X}$, et al. SEOM Clinical Guidelines for Diagnosis and Treatment of Glioblastoma (2017). Clin Trans Oncol Off Publ Fed Spanish Oncol Soc Natl Cancer Inst Mexico (2018) 20(1):22-8. doi: 10.1007/s12094-017-1763-6

7. Stupp R, Mason WP, van den Bent MJ, Weller M, Fisher B, Taphoorn MJ, et al. Radiotherapy Plus Concomitant and Adjuvant Temozolomide for Glioblastoma. N Engl J Med (2005) 352(10):987-96. doi: 10.1056/ NEJMoa043330

8. Sanai N, Berger MS. Surgical Oncology for Gliomas: The State of the Art. Nat Rev Clin Oncol (2018) 15(2):112-25. doi: 10.1038/nrclinonc.2017.171

9. Wang Y, Jiang T. Understanding High Grade Glioma: Molecular Mechanism, Therapy and Comprehensive Management. Cancer Lett (2013) 331(2):13946. doi: 10.1016/j.canlet.2012.12.024

10. Khan MN, Sharma AM, Pitz M, Loewen SK, Quon H, Poulin A, et al. HighGrade Glioma Management and Response Assessment-Recent Advances and Current Challenges. Curr Oncol (2016) 23(4):e383-91. doi: 10.3747/ co.23.3082

11. Molinaro AM, Hervey-Jumper S, Morshed RA, Young J, Han SJ, Chunduru P, et al. Association of Maximal Extent of Resection of Contrast-Enhanced and Non-Contrast-Enhanced Tumor With Survival Within Molecular Subgroups of Patients With Newly Diagnosed Glioblastoma. JAMA Oncol (2020) 6 (4):495-503. doi: 10.1001/jamaoncol.2019.6143

12. Brown PD, Maurer MJ, Rummans TA, Pollock BE, Ballman KV, Sloan JA, et al. A Prospective Study of Quality of Life in Adults With Newly Diagnosed High-Grade Gliomas: The Impact of the Extent of Resection on Quality of Life and Survival. Neurosurgery (2005) 57(3):495-504; discussion 495-504. doi: 10.1227/ 01.neu.0000170562.25335.c7

13. Haj A, Doenitz C, Schebesch KM, Ehrensberger D, Hau P, Putnik K, et al. Extent of Resection in Newly Diagnosed Glioblastoma: Impact of a Specialized Neuro-Oncology Care Center. Brain Sci (2017) 8(1):5. doi: 10.3390/ brainsci8010005

14. Ammirati M, Vick N, Liao YL, Ciric I, Mikhael M. Effect of the Extent of Surgical Resection on Survival and Quality of Life in Patients With Supratentorial Glioblastomas and Anaplastic Astrocytomas. Neurosurgery (1987) 21(2):201-6. doi: 10.1227/00006123-198708000-00012

\section{ACKNOWLEDGMENTS}

We would like to thank Gabriel Girard for discussion and advice during analyses of data for this study.

\section{SUPPLEMENTARY MATERIAL}

The Supplementary Material for this article can be found online at: https://www.frontiersin.org/articles/10.3389/fonc.2021.761169/ full\#supplementary-material

15. Hervey-Jumper SL, Berger MS. Evidence for Improving Outcome Through Extent of Resection. Neurosurg Clin N Am (2019) 30(1):85-93. doi: 10.1016/ j.nec.2018.08.005

16. Lacroix M, Abi-Said D, Fourney DR, Gokaslan ZL, Shi W, DeMonte F, et al. A Multivariate Analysis of 416 Patients With Glioblastoma Multiforme: Prognosis, Extent of Resection, and Survival. J Neurosurg (2001) 95(2):1908. doi: 10.3171/jns.2001.95.2.0190

17. Bloch O, Han SJ, Cha S, Sun MZ, Aghi MK, McDermott MW, et al. Impact of Extent of Resection for Recurrent Glioblastoma on Overall Survival: Clinical Article. J Neurosurg (2012) 117(6):1032-8. doi: 10.3171/2012.9.JNS12504

18. Duffau H, Mandonnet E. The "Onco-Functional Balance" in Surgery for Diffuse Low-Grade Glioma: Integrating the Extent of Resection With Quality of Life. Acta Neurochirurgica (2013) 155(6):951-7. doi: 10.1007/s00701-0131653-9

19. Bush NA, Chang SM, Berger MS. Current and Future Strategies for Treatment of Glioma. Neurosurg Rev (2016) 40:1-14. doi: 10.1007/s10143-016-0709-8

20. Sanai N, Berger MS. Intraoperative Stimulation Techniques for Functional Pathway Preservation and Glioma Resection. Neurosurg Focus (2010) 28(2): E1. doi: 10.3171/2009.12.FOCUS09266

21. De Witt Hamer PC, Robles SG, Zwinderman AH, Duffau H, Berger MS. Impact of Intraoperative Stimulation Brain Mapping on Glioma Surgery Outcome: A Meta-Analysis. J Clin Oncol Off J Am Soc Clin Oncol (2012) 30 (20):2559-65. doi: 10.1200/JCO.2011.38.4818

22. Hervey-Jumper SL, Berger MS. Maximizing Safe Resection of Low- and HighGrade Glioma. J Neuro-Oncol (2016) 130(2):269-82. doi: 10.1007/s11060-0162110-4

23. Villanueva-Meyer JE, Mabray MC, Cha S. Current Clinical Brain Tumor Imaging. Neurosurgery (2017) 81(3):397-415. doi: 10.1093/neuros/nyx103

24. Shukla G, Alexander GS, Bakas S, Nikam R, Talekar K, Palmer JD, et al. Advanced Magnetic Resonance Imaging in Glioblastoma: A Review. Chin Clin Oncol (2017) 6(4):40. doi: 10.21037/cco.2017.06.28

25. Overcast WB, Davis KM, Ho CY, Hutchins GD, Green MA, Graner BD, et al. Advanced Imaging Techniques for Neuro-Oncologic Tumor Diagnosis, With an Emphasis on PET-MRI Imaging of Malignant Brain Tumors. Curr Oncol Rep (2021) 23(3):34. doi: 10.1007/s11912-021-01020-2

26. Smits M. MRI Biomarkers in Neuro-Oncology. Nat Rev Neurol (2021) 17:486-500. doi: 10.1038/s41582-021-00510-y

27. Assaf Y, Pasternak O. Diffusion Tensor Imaging (DTI)-Based White Matter Mapping in Brain Research: A Review. J Mol Neurosci (2008) 34(1):51-61. doi: 10.1007/s12031-007-0029-0

28. Ulmer JL, Klein AP, Mueller WM, DeYoe EA, Mark LP. Preoperative Diffusion Tensor Imaging: Improving Neurosurgical Outcomes in Brain Tumor Patients. Neuroimaging Clinics N Am (2014) 24(4):599-617. doi: 10.1016/j.nic.2014.08.002

29. Ulmer JL, Salvan CV, Mueller WM, Krouwer HG, Stroe GO, Aralasmak A, et al. The Role of Diffusion Tensor Imaging in Establishing the Proximity of Tumor Borders to Functional Brain Systems: Implications for Preoperative Risk Assessments and Postoperative Outcomes. Technol Cancer Res Treat (2004) 3(6):567-76. doi: 10.1177/153303460400300606

30. Basser PJ, Mattiello J, LeBihan D. MR Diffusion Tensor Spectroscopy and Imaging. Biophys J (1994) 66(1):259-67. doi: 10.1016/S0006-3495(94)80775-1 
31. Abdullah KG, Lubelski D, Nucifora PG, Brem S. Use of Diffusion Tensor Imaging in Glioma Resection. Neurosurgical Focus (2013) 34(4):E1. doi: 10.3171/2013.1.FOCUS12412

32. Maier-Hein KH, Neher PF, Houde JC, Cote MA, Garyfallidis E, Zhong J, et al. The Challenge of Mapping the Human Connectome Based on Diffusion Tractography. Nat Commun (2017) 8(1):1349. doi: 10.1038/s41467-01701285-x

33. Farquharson S, Tournier JD, Calamante F, Fabinyi G, Schneider-Kolsky M, Jackson GD, et al. White Matter Fiber Tractography: Why We Need to Move Beyond DTI. J Neurosurg (2013) 118(6):1367-77. doi: 10.3171/2013.2. JNS121294

34. Nimsky C. Fiber Tracking-A Reliable Tool for Neurosurgery? World Neurosurg (2010) 74(1):105-6. doi: 10.1016/j.wneu.2010.05.014

35. Duffau H. Diffusion Tensor Imaging Is a Research and Educational Tool, But Not Yet a Clinical Tool. World Neurosurg (2014) 82(1-2):e43-5. doi: 10.1016/ j.wneu.2013.08.054

36. Potgieser AR, Wagemakers $M$, van Hulzen AL, de Jong BM, Hoving EW, Groen RJ. The Role of Diffusion Tensor Imaging in Brain Tumor Surgery: A Review of the Literature. Clin Neurol Neurosurg (2014) 124:51-8. doi: 10.1016/j.clineuro.2014.06.009

37. Becker D, Scherer M, Neher P, Jungk C, Jesser J, Pfluger I, et al. Going Beyond Diffusion Tensor Imaging Tractography in Eloquent Glioma Surgery-HighResolution Fiber Tractography: Q-Ball or Constrained Spherical Deconvolution? World Neurosurg (2020) 134:e596-609. doi: 10.1016/j.wneu.2019.10.138

38. Behrens TE, Berg HJ, Jbabdi S, Rushworth MF, Woolrich MW. Probabilistic Diffusion Tractography With Multiple Fibre Orientations: What Can We Gain? NeuroImage (2007) 34(1):144-55. doi: 10.1016/j.neuroimage. 2006.09.018

39. Jeurissen B, Leemans A, Tournier JD, Jones DK, Sijbers J. Investigating the Prevalence of Complex Fiber Configurations in White Matter Tissue With Diffusion Magnetic Resonance Imaging. Hum Brain Mapp (2013) 34 (11):2747-66. doi: 10.1002/hbm.22099

40. Jensen JH, Helpern JA, Ramani A, Lu H, Kaczynski K. Diffusional Kurtosis Imaging: The Quantification of Non-Gaussian Water Diffusion by Means of Magnetic Resonance Imaging. Magnetic Reson Med Off J Soc Magnetic Reson Med / Soc Magnetic Reson Med (2005) 53(6):1432-40. doi: 10.1002/ mrm.20508

41. Tournier JD, Calamante F, Connelly A. Robust Determination of the Fibre Orientation Distribution in Diffusion MRI: Non-Negativity Constrained Super-Resolved Spherical Deconvolution. NeuroImage (2007) 35(4):145972. doi: 10.1016/j.neuroimage.2007.02.016

42. Jeurissen B, Leemans A, Jones DK, Tournier JD, Sijbers J. Probabilistic Fiber Tracking Using the Residual Bootstrap With Constrained Spherical Deconvolution. Hum Brain Mapp (2011) 32(3):461-79. doi: 10.1002/ hbm. 21032

43. Mortazavi F, Oblak AL, Morrison WZ, Schmahmann JD, Stanley HE, Wedeen VJ, et al. Geometric Navigation of Axons in a Cerebral Pathway: Comparing dMRI With Tract Tracing and Immunohistochemistry. Cereb Cortex (2018) 28(4):1219-32. doi: 10.1093/cercor/bhx034

44. Mormina E, Longo M, Arrigo A, Alafaci C, Tomasello F, Calamuneri A, et al. MRI Tractography of Corticospinal Tract and Arcuate Fasciculus in HighGrade Gliomas Performed by Constrained Spherical Deconvolution: Qualitative and Quantitative Analysis. AJNR Am J Neuroradiol (2015) 36 (10):1853-8. doi: 10.3174/ajnr.A4368

45. Sheng Z, Yu J, Chen Z, Sun Y, Bu X, Wang M, et al. Constrained-Spherical Deconvolution Tractography in the Evaluation of the Corticospinal Tract in Glioma Surgery. Front Surg (2021) 8:646465. doi: 10.3389/fsurg.2021.646465

46. Mormina E, Arrigo A, Calamuneri A, Alafaci C, Tomasello F, Morabito R, et al. Optic Radiations Evaluation in Patients Affected by High-Grade Gliomas: A Side-by-Side Constrained Spherical Deconvolution and Diffusion Tensor Imaging Study. Neuroradiology (2016) 58(11):1067-75. doi: 10.1007/s00234-016-1732-8

47. Tournier JD, Smith R, Raffelt D, Tabbara R, Dhollander T, Pietsch M, et al. MRtrix3: A Fast, Flexible and Open Software Framework for Medical Image Processing and Visualisation. NeuroImage (2019) 202:116137. doi: 10.1016/ j.neuroimage.2019.116137

48. Zhylka A, Leemans A, Pluim J, De Luca A. Anatomically Informed MultiLevel Fiber Tractography. bioRxiv (2020). doi: 10.1101/2020.12.16.423042.
49. Zhylka A, Leemans A, Pluim J, De Luca A. Anatomically Informed MultiLevel Fiber Tractography for Improved Sensitivity of White Matter Bundle Reconstruction in Diffusion MRI. Proc Intl Soc Mag Reson Med (2020) 28:855.

50. Radwan AM, Emsell L, Blommaert J, Zhylka A, Kovacs S, Theys T, et al. Virtual Brain Grafting: Enabling Whole Brain Parcellation in the Presence of Large Lesions. NeuroImage (2021) 229:117731. doi: 10.1016/ j.neuroimage.2021.117731

51. Fischl B. FreeSurfer. NeuroImage (2012) 62(2):774-81. doi: 10.1016 j.neuroimage.2012.01.021

52. Kofler F, Berger C, Waldmannstetter D, Lipkova J, Ezhov I, Tetteh G, et al. BraTS Toolkit: Translating BraTS Brain Tumor Segmentation Algorithms Into Clinical and Scientific Practice. Front Neurosci (2020) 14:125. doi: 10.3389/fnins.2020.00125

53. Menze BH, Jakab A, Bauer S, Kalpathy-Cramer J, Farahani K, Kirby J, et al. The Multimodal Brain Tumor Image Segmentation Benchmark (BRATS). IEEE Trans Med Imaging (2015) 34(10):1993-2024. doi: 10.1109/ TMI.2014.2377694

54. Leemans A, Jeurissen B, Sijbers J, Jones DK. ExploreDTI: A Graphical Toolbox for Processing, Analyzing, and Visualizing Diffusion MR Data. Proc Intl Soc Mag Reson Med (2009) 17:3537.

55. Tax CM, Jeurissen B, Vos SB, Viergever MA, Leemans A. Recursive Calibration of the Fiber Response Function for Spherical Deconvolution of Diffusion MRI Data. NeuroImage (2014) 86:67-80. doi: 10.1016/ j.neuroimage.2013.07.067

56. Desikan RS, Segonne F, Fischl B, Quinn BT, Dickerson BC, Blacker D, et al. An Automated Labeling System for Subdividing the Human Cerebral Cortex on MRI Scans Into Gyral Based Regions of Interest. NeuroImage (2006) 31 (3):968-80. doi: 10.1016/j.neuroimage.2006.01.021

57. Costabile JD, Alaswad E, D'Souza S, Thompson JA, Ormond DR. Current Applications of Diffusion Tensor Imaging and Tractography in Intracranial Tumor Resection. Front Oncol (2019) 9:426. doi: 10.3389/fonc.2019.00426

58. Weiss C, Tursunova I, Neuschmelting V, Lockau H, Nettekoven C, OrosPeusquens AM, et al. Improved nTMS- and DTI-Derived CST Tractography Through Anatomical ROI Seeding on Anterior Pontine Level Compared to Internal Capsule. NeuroImage Clin (2015) 7:424-37. doi: 10.1016/ j.nicl.2015.01.006

59. Jellison BJ, Field AS, Medow J, Lazar M, Salamat MS, Alexander AL. Diffusion Tensor Imaging of Cerebral White Matter: A Pictorial Review of Physics, Fiber Tract Anatomy, and Tumor Imaging Patterns. AJNR Am J Neuroradiol (2004) 25(3):356-69.

60. Virtanen P, Gommers R, Oliphant TE, Haberland M, Reddy T, Cournapeau D, et al. SciPy 1.0: Fundamental Algorithms for Scientific Computing in Python. Nat Methods (2020) 17(3):261-72. doi: 10.1038/s41592-019-0686-2

61. Tuch DS. Q-Ball Imaging. Magnetic Reson Med Off J Soc Magnetic Reson Med / Soc Magnetic Reson Med (2004) 52(6):1358-72. doi: 10.1002/mrm.20279

62. Bucci M, Mandelli ML, Berman JI, Amirbekian B, Nguyen C, Berger MS, et al. Quantifying Diffusion MRI Tractography of the Corticospinal Tract in Brain Tumors With Deterministic and Probabilistic Methods. NeuroImage Clin (2013) 3:361-8. doi: 10.1016/j.nicl.2013.08.008

63. Tournier JD, Calamante F, Gadian DG, Connelly A. Direct Estimation of the Fiber Orientation Density Function From Diffusion-Weighted MRI Data Using Spherical Deconvolution. NeuroImage (2004) 23(3):1176-85. doi: 10.1016/j.neuroimage.2004.07.037

64. Conturo TE, Lori NF, Cull TS, Akbudak E, Snyder AZ, Shimony JS, et al. Tracking Neuronal Fiber Pathways in the Living Human Brain. Proc Natl Acad Sci USA (1999) 96(18):10422-7. doi: 10.1073/pnas.96.18.10422

65. Guye M, Parker GJ, Symms M, Boulby P, Wheeler-Kingshott CA, SalekHaddadi A, et al. Combined Functional MRI and Tractography to Demonstrate the Connectivity of the Human Primary Motor Cortex In Vivo. NeuroImage (2003) 19(4):1349-60. doi: 10.1016/s1053-8119(03) 00165-4

66. Staempfli P, Reischauer C, Jaermann T, Valavanis A, Kollias S, Boesiger P. Combining fMRI and DTI: A Framework for Exploring the Limits of fMRIGuided DTI Fiber Tracking and for Verifying DTI-Based Fiber Tractography Results. NeuroImage (2008) 39(1):119-26. doi: 10.1016/j.neuroimage. 2007.08.025

67. Sollmann N, Wildschuetz N, Kelm A, Conway N, Moser T, Bulubas L, et al. Associations Between Clinical Outcome and Navigated Transcranial Magnetic 
Stimulation Characteristics in Patients With Motor-Eloquent Brain Lesions: A Combined Navigated Transcranial Magnetic Stimulation-Diffusion Tensor Imaging Fiber Tracking Approach. J Neurosurg (2018) 128(3):800-10. doi: $10.3171 / 2016.11$.JNS162322

68. Sollmann N, Zhang H, Fratini A, Wildschuetz N, Ille S, Schroder A, et al. Risk Assessment by Presurgical Tractography Using Navigated TMS Maps in Patients With Highly Motor- or Language-Eloquent Brain Tumors. Cancers (Basel) (2020) 12(5):1264. doi: 10.3390/cancers12051264

69. Frey D, Strack V, Wiener E, Jussen D, Vajkoczy P, Picht T. A New Approach for Corticospinal Tract Reconstruction Based on Navigated Transcranial Stimulation and Standardized Fractional Anisotropy Values. NeuroImage (2012) 62(3):1600-9. doi: 10.1016/j.neuroimage.2012.05.059

70. Krieg SM, Buchmann NH, Gempt J, Shiban E, Meyer B, Ringel F. Diffusion Tensor Imaging Fiber Tracking Using Navigated Brain Stimulation-a Feasibility Study. Acta Neurochirurgica (2012) 154(3):555-63. doi: 10.1007/ s00701-011-1255-3

71. Weiss Lucas C, Tursunova I, Neuschmelting V, Nettekoven C, OrosPeusquens AM, Stoffels G, et al. Functional MRI vs. Navigated TMS to Optimize M1 Seed Volume Delineation for DTI Tractography. A Prospective Study in Patients With Brain Tumours Adjacent to the Corticospinal Tract. NeuroImage Clin (2017) 13:297-309. doi: 10.1016/j.nicl.2016.11.022

72. Sollmann N, Meyer B, Krieg SM. Implementing Functional Preoperative Mapping in the Clinical Routine of a Neurosurgical Department: Technical Note. World Neurosurg (2017) 103:94-105. doi: 10.1016/j.wneu. 2017.03.114

73. Sollmann N, Fratini A, Zhang H, Zimmer C, Meyer B, Krieg SM. Associations Between Clinical Outcome and Tractography Based on Navigated Transcranial Magnetic Stimulation in Patients With Language-Eloquent Brain Lesions. J neurosurg (2019) 132(4):1033-42. doi: 10.3171/2018.12.JNS182988

74. Sollmann N, Negwer C, Ille S, Maurer S, Hauck T, Kirschke JS, et al. Feasibility of nTMS-Based DTI Fiber Tracking of Language Pathways in Neurosurgical Patients Using a Fractional Anisotropy Threshold. J Neurosci Methods (2016) 267:45-54. doi: 10.1016/j.jneumeth.2016.04.002
75. Raffa G, Bahrend I, Schneider H, Faust K, Germano A, Vajkoczy P, et al. A Novel Technique for Region and Linguistic Specific nTMS-Based DTI Fiber Tracking of Language Pathways in Brain Tumor Patients. Front Neurosci (2016) 10:552. doi: 10.3389/fnins.2016.00552

76. De Luca A, Guo F, Froeling M, Leemans A. Spherical Deconvolution With Tissue-Specific Response Functions and Multi-Shell Diffusion MRI to Estimate Multiple Fiber Orientation Distributions (mFODs). NeuroImage (2020) 222:117206. doi: 10.1016/j.neuroimage.2020.117206

Conflict of Interest: NS received honoraria from Nexstim Plc (Helsinki, Finland) and Philips Healthcare (Best, The Netherlands). SK is consultant for Ulrich medical (Ulm, Germany) and Brainlab AG (Munich, Germany) and received honoraria from Nexstim Plc (Helsinki, Finland), Spineart Deutschland GmbH (Frankfurt, Germany), Medtronic (Meerbusch, Germany) and Carl Zeiss Meditec (Oberkochen, Germany).

The remaining authors declare that the research was conducted in the absence of any commercial or financial relationships that could be construed as a potential conflict of interest.

Publisher's Note: All claims expressed in this article are solely those of the authors and do not necessarily represent those of their affiliated organizations, or those of the publisher, the editors and the reviewers. Any product that may be evaluated in this article, or claim that may be made by its manufacturer, is not guaranteed or endorsed by the publisher.

Copyright (c) 2021 Zhylka, Sollmann, Kofler, Radwan, De Luca, Gempt, Wiestler, Menze, Krieg, Zimmer, Kirschke, Sunaert, Leemans and Pluim. This is an open-access article distributed under the terms of the Creative Commons Attribution License (CC BY). The use, distribution or reproduction in other forums is permitted, provided the original author(s) and the copyright owner(s) are credited and that the original publication in this journal is cited, in accordance with accepted academic practice. No use, distribution or reproduction is permitted which does not comply with these terms. 